\title{
BODY COMPOSITION USING BIO-IMPEDANCE ANALYSIS IN PEDIATRIC PATIENTS WITH INFLAMMATORY BOWEL DISEASE. CONCORDANCE WITH DUAL ENERGY X-RAY ABSORPTIOMETRY AND COMPARISON WITH HEALTHY CONTROLS
}

C. Callias ${ }^{1}$, T. Chatton ${ }^{1}$, P.M. Marques - Vidal ${ }^{2}$, J. Ezri ${ }^{1}$, A. Nydegger ${ }^{1}$

$1^{1}$ Pediatric Gastroenterology, Hepatology and Nutrition ${ }^{2}$ Institute of social and Preventive Medicine (IUMSP) University Hospital Lausanne, Lausanne, Switzerland

FNSWF

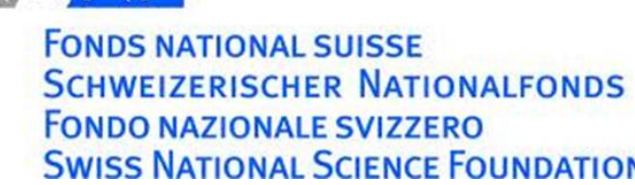

\section{BACKGROUND:}

Growth is a central process in paediatrics. Weight and height evaluation are therefore routine exams for every child but in some situation, particularly inflammatory bowel disease (IBD), a wider evaluation of nutritional status needs to be performed.

Twenty-five percent of Crohn's diseases (CD) are diagnosed during puberty, with growth failure often being the predominant initial manifestation.

The underlying mechanisms of growth retardation are not fully understood but may be primarily related to malnutrition and to the strong inflammatory reaction occurring during active disease.
OBJECTIVES: 1 . Assess the accuracy of BIA in estimating body composition (percentage fat mass: FM\% and percentage fat free mass: FFM\%) in children with IBD, compared with DEXA (gold standard)

2. To compare $\mathrm{FM} \%$ and $\mathrm{FFM} \%$ levels between IBD patients and healthy controls

\section{PATIENTS AND METHOD: (figure 1)}

21 patients with inflammatory bowel disease were assessed (11 females, 10 males, 15 with Crohn's disease and 6 with ulcerative colitis). Mean age was 14.8 years (range 12-16 years). The patients were compared to a group of 29 healthy controls (12 females, 17 males). Mean age was 12.7 years (range 10-16 years).

The patients were recruited from August 2011 to October 2012 at our institution.

In both groups, BIA using BIA 101 - Body impedance analyser (AKERN, Florence, Italy) was performed. This technique is based on the properties of tissues to conduct electrical current. Using values of TBW derived from BIA, one can then estimate fat-free mass (FFM) and body fat (adiposity).

BodyGram Pro ${ }^{\circledast}$ is a multi-functional BIA software for medically validated analysis of body composition and hydration covering both sexes, all age ranges and constitutional types. The equations used are protected by the manufacturer but probably look like this (ref. 1-3)

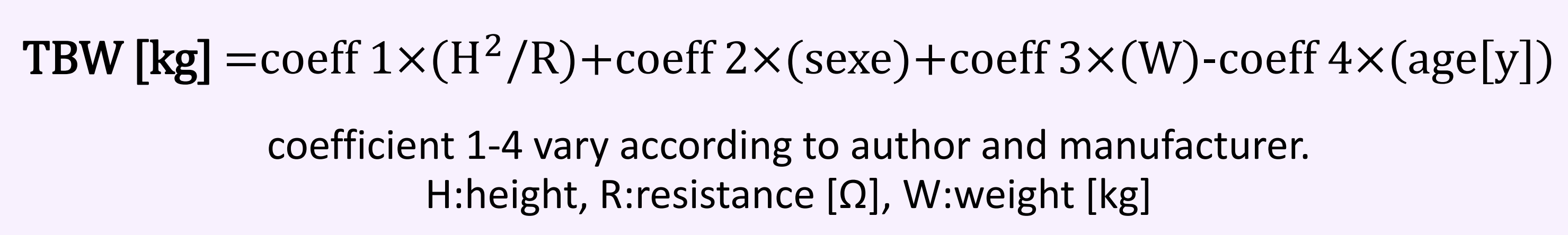

FFM $[\mathrm{kg}]=\mathrm{TBW} /(0.73) \quad$ and $\quad \mathrm{FM}[\mathrm{kg}]=$ Weight $[\mathrm{kg}]-\mathrm{FFM}$

BIA was performed in all children and DEXA in patients only. Concordance between BIA and DEXA was assessed using Lin's concordance correlation and the Bland-Altman method. Between-group comparisons were made using analysis of variance adjusting for age.

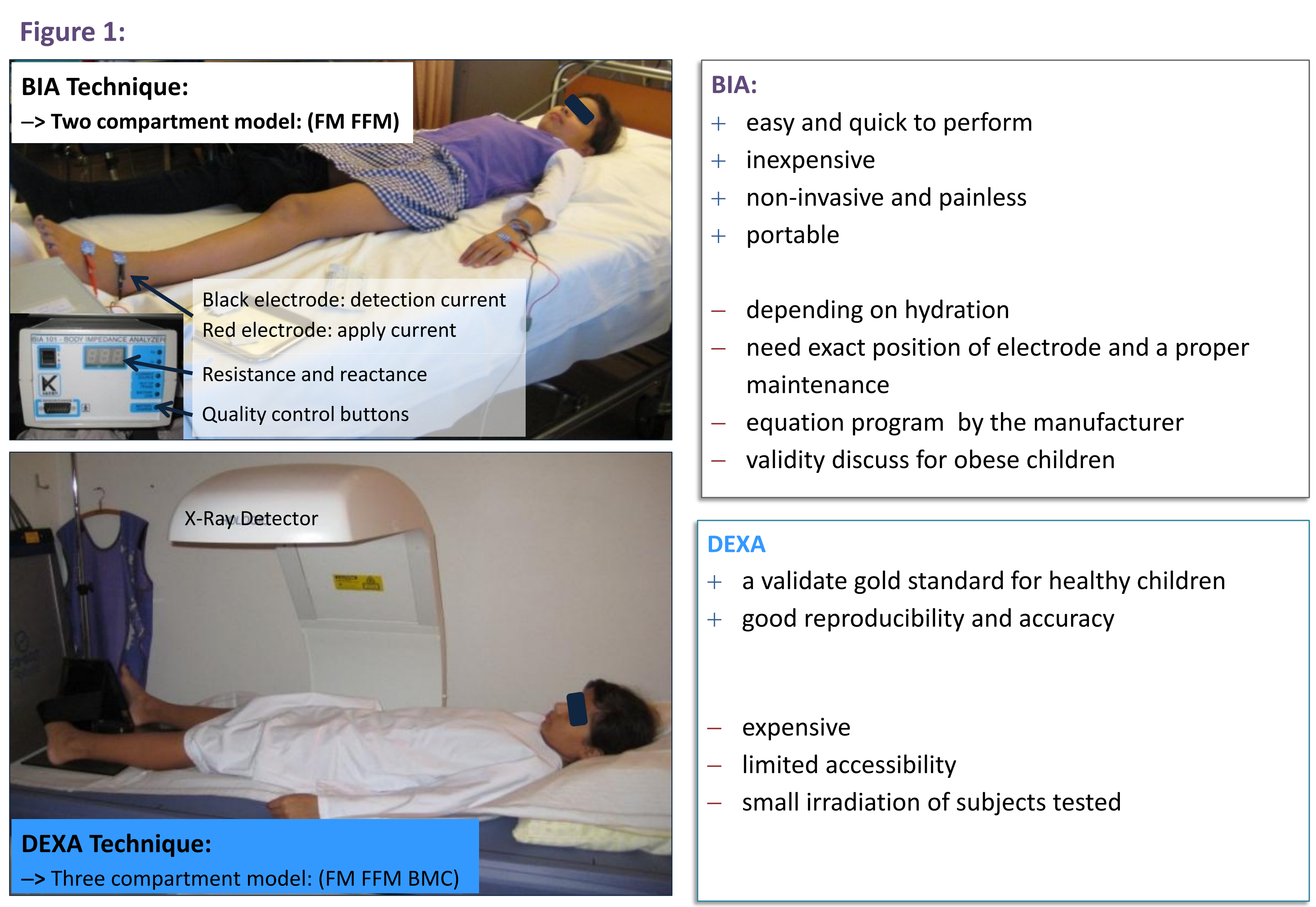

\section{BIBLIOGRAPHY:}

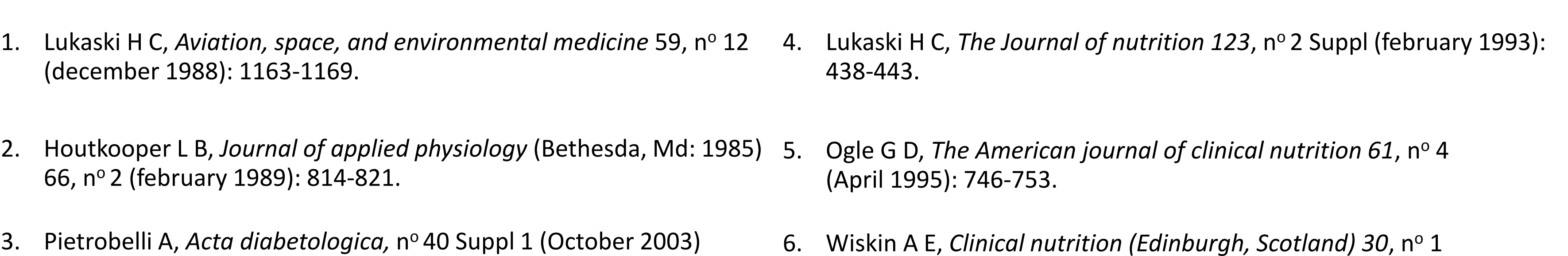

Assessment of body composition therefore is crucial in order to maintain acceptable growth using the following techniques: Dual-energy-x-ray absorptiometry (DEXA), bioimpedance-analysis (BIA) and anthropometric measurements (skinfold thickness).

Even though DEXA is known as the gold standard for body composition assessment, its availability, invasiveness and relatively high cost renders it rather impractical. Therefore a technique like BIA showing the simplicity of skinfold measurements and the precision of DEXA in the same time could be used as a bedside tool. 